\title{
SÍNDROME DE BECKWITH WIEDEMANN BECKWITH WIEDEMANN SYNDROME SÍNDROME DE BECKWITH WIEDEMANN
}

\section{RUTH MARGARITA GUEVARA BARRERA}

Universidad Católica de Santiago de Guayaquil, Guayaquil, Ecuador

\section{Resumen}

El Síndrome de Beckwith Wiedemann, es una enfermedad de origen genético autosómico dominante, caracterizado por macrosomía, onfalocele, dismorfismo cráneo-facial, macroglosia, visceromegalia, hemihipertrofia y tumores como el de Wilms. El diagnóstico se fundamenta en criterios clínicos ( criterios mayores y menores ). El mismo se realiza con la asociación de los tres criterios mayores o de dos criterios mayores y tres menores. (2) Presentación de caso: a continuación se presenta el caso clínico de un paciente de género masculino de 4 horas de nacido atendido en el Servicio de Neonatología del hospital del niño Francisco de Ycaza Bustamante de la ciudad de (Guayaquil), portador del Síndrome de Beckwith Wiedemann. Se exponen los resultados del examen clínico y de las investigaciones complementarias.

PALABRAS CLAVE: onfalocele, macroglosia, hipoglucemia, hemihipertrofia, nevus flammeus facial.

Abstract

Beckwith Wiedemann syndrome is a disease of autosomal dominant genetic origin, characterized by macrosomia, omphalocele, craniofacial dysmorphism, macroglossia, visceromegaly, hemihypertrophy and tumors such as Wilms tumor. The diagnosis is based on clinical criteria (major and minor criteria). This is done with the association of three major criteria or two major criteria and three minor ones. (2) Case presentation: This is the clinical case of a 4-hour-old male patient seen at the Neonatology Service of the Francisco de Ycaza Bustamante Hospital of the city of (Guayaquil), which presents with the Beckwith Wiedemann syndrome. The results of the clinical examination and the complementary investigations are exposed.

KEYWORDS: omphalocele, macroglossia, hypoglycemia, hemihypertrophy, nevus flammeus facial.

Resumo

A síndrome de Beckwith Wiedemann é uma doença de origem genética autossômica dominante, caracterizada por macrossomia, onfalocele, dismorfismo craniofacial, macroglossia, visceromegalia, hemi-hipertrofia e tumores como o Wilms. 0 diagnóstico é baseado em critérios clínicos (critérios maiores e menores). 0 mesmo é feito com a associação dos três critérios principais ou dois critérios principais e três menores. (2) Apresentação do caso: 0 caso clínico de um paciente do sexo masculino de 4 horas de idade visto no Serviço de Neonatologia do Hospital Francisco del Ycaza Bustamante da cidade de Guayaquil, portador da Síndrome, é apresentado abaixo. de Beckwith Wiedemann. Os resultados do exame clínico e das investigações complementares estão expostos.

PALAVRAS-CHAVE: onfalocele, macroglossia, hipoglicemia, hemi-hipertrofia, nevo flammeus facial. 
INTRODUCCIÓN

El síndrome de Beckwith-Wiedemann (SBW) es una enfermedad genética, descrita por primera vez en 1963; es uno de los síndromes congénitos más frecuentes, ${ }^{1,6}$ con hiper-crecimiento, macroglosia, onfalocele, anomalías umbilicales, hipoglicemia sintomática. ${ }^{2}$ las manifestaciones en cabeza y cuello son de expresión variable. ${ }^{3}$

Puede presentarse aproximadamente en 1 de cada 14000 nacimientos (20), aunque es posible que no sean diagnosticados los casos con manifestaciones clínicas menos notorias. La incidencia en sexo femenino y masculino es similar y la frecuencia de presentación es igual en individuos de raza blanca o negra. Está establecido la localización de los genes causantes en el cromosoma 11 (11p15.5). ${ }^{3-7}$ Su diagnóstico es importante por el riesgo asociado de desarrollo de tumores embrionarios, además tiene un patrón de herencia que afecta morfológicamente al paciente y pone en riesgo la vida. ${ }^{6}$

\section{CASO CLÍNICO}

Se presenta el caso de un niño de 4 horas de vida, sexo masculino, peso $4 \mathrm{~kg}$ superior a P90, en el Servicio de Neonatología del hospital del niño Francisco de Ycaza Bustamante procedente de la Maternidad Mariana de Jesús por onfalocele, macroglosia, macrosómico, hipoglucemia, siendo tratado con fluido terapia, antibioticoterapia, terapia del dolor, intervención quirúrgica.

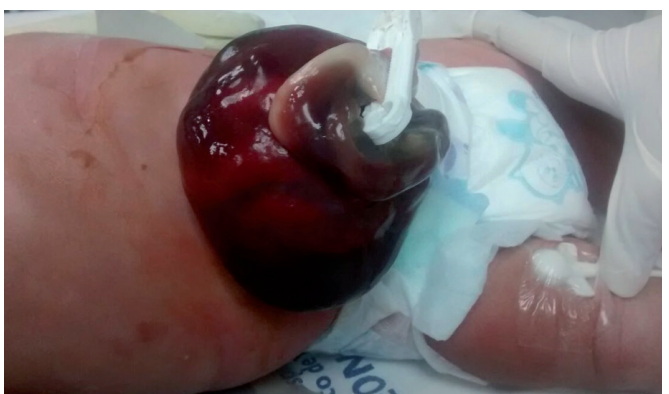

Figura 1. Onfalocele, defecto de pared abdominal central, a nivel del anillo y cordón umbilical, cubierto por una membrana formada por amnios con gelatina de Wharton. ${ }^{8,9}$

Nace por parto distócico, a las 40 semanas de gestación con Apgar de 8 - 9, sin antecedentes paternos de importancia; gestas previas 2 sin ninguna anomalía; no abortos; controles prenatales por 7 ocasiones, sin exposición materna a ningún factor externo; existe consanguinidad entre los padres.
Al examen físico: Producto macrosómico, fascie dismórfica, macrocefálico, con fontanelas anterior, amplia; y posterior, abierta; suturas cabalgadas, macroglosia y cuello corto (Figura 2).

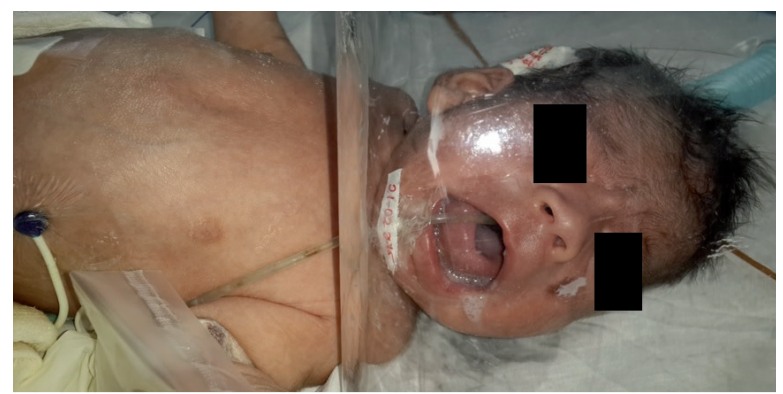

Figura 2. Paciente masculino de 3 días de vida; se observa macroglosia. ${ }^{10,16}$

A la auscultación ruidos cardiacos rítmicos sin soplos agregados; campos pulmonares ventilados; murmullo vesicular audible; abdomen con presencia de defecto de pared de tipo onfalocele. (Figura 1), extremidades superiores e inferiores simétricas.

Se decide resolución quirúrgica. El paciente fue intervenido por 2 ocasiones para reducción de asas a través del defecto del cordón umbilical (Figura 3). Recibió esquema antibiótico con oxacilina $200 \mathrm{mg} / \mathrm{kg} /$ día -amikacina $15 \mathrm{mg} / \mathrm{kg} /$ día por 10 días por la manipulación quirúrgica así como también por aumento de reactantes de fase aguda: leucocitosis $20300, \mathrm{~mm} 3$ neutrofilia $76.6 \%$, procalcitonina $18 \mathrm{ng} / \mathrm{ml}$.

Recibió terapia del dolor con paracetamol 15mg / $\mathrm{kg} /$ dosis intravenoso. Permaneció hospitalizado 15 días; buena respuesta clínica, tolerando la vía oral.

Resultados de laboratorio: leucocitos $11200 \mathrm{~mm} 3$, linfocitos $22,3 \%$, neutrófilos $70 \%$ hcto $50 \%$ hb $16,6 \mathrm{~g} / \mathrm{dl}$, plaquetas $250000 \mathrm{~mm} 3$, PCR 1,8mg/dl; por su buena evolución clínica es dado de alta.

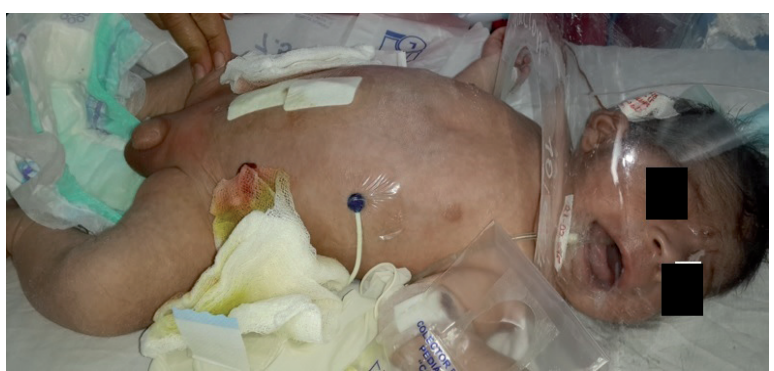

Figura 3. Neonato masculino de 5 días de vida, posoperatorio: reducción de onfalocele, mal rotación completa de asas intestinales, laparoplastia, cierre de ileostomía, yeyustomía. 
EXÁMENES DE LABORATORIO

\begin{tabular}{lrr} 
BHC & INGRESO & EGRESO \\
\hline LEUCO & $20300^{*}$ & 11.2 \\
\hline HCT & $54,3 \%$ & $50 \%$ \\
\hline HB & $17.3 \mathrm{~g}$. & $16.6 \mathrm{~g}$. \\
\hline GR & 5.76 & 5.37 \\
\hline SEG & $77.6 \% \%^{*}$ & $70 \%$ \\
\hline LIN & $15.6 \%$ & $22,3 \%$ \\
\hline MON & $6.2 \%$ & $4,6 \%$ \\
\hline PLAQ & 360000 & 250000 \\
\hline PCR & $0.3 \mathrm{mg} / \mathrm{L}$ & $1.8 \mathrm{mg} / \mathrm{L}$ \\
\hline VDRL & $\mathrm{NO} \mathrm{REACTIV0}$ & \\
\hline CA & $9.3 \mathrm{mg} / \mathrm{dL}$ & 10,4 \\
\hline GLUCOSA & $50 \mathrm{~g} . \mathrm{mg} / \mathrm{dL}$ & $89 \mathrm{mg} / \mathrm{dL}$ \\
\hline NA & $135 \mathrm{mEq} / \mathrm{L}$ & $137 \mathrm{meq}$ \\
\hline K & $4 \mathrm{meq} \mathrm{mEq} / \mathrm{L}$ & $4,5 \mathrm{meq}$ \\
\hline Proteinas totales & $5,8 \mathrm{~g}$. & $5,7 \mathrm{~g}$. \\
\hline Albumina & $3 \mathrm{~g}$. & $3.5 \mathrm{~g}$. \\
\hline TGO & 58 & 60 \\
\hline TGP & 24 & 23 \\
\hline Procalcitonina & $18 \mathrm{mg} / \mathrm{mL}$ & $0,5 \mathrm{mg} / \mathrm{mL}$ \\
\hline *Factor reactantes de fase aguda, e hipoglicemia &
\end{tabular}

DISCUSIÓN

El Síndrome de Beckwith-Wiedemann es una entidad clínica subdiagnosticada debido a que algunos casos de menor severidad, pueden confundirse con defectos simples de pared abdominal o niños grandes para la edad gestacional, ${ }^{18}$ genéticamente en este síndrome, se han descrito tres formas de herencia, a saber: la esporádica, la familiar y las alteraciones cromosómicas. En la primera forma de herencia no se ha establecido riesgo de recurrencia, para la segunda se describe un patrón autosómico dominante que puede presentar penetrancia incompleta y expresividad variable. En los dos primeros casos no hay alteraciones citogenéticas a diferencia de la en la que se pueden encontrar duplicaciones del cromosoma 11p15.5, inversiones en el 11p distal, o translocaciones balanceadas que rompen esta región. ${ }^{19}$

Se tienen datos de una prevalencia de 1/14000 en la población en general, ${ }^{20}$ aunque dado que existen fenotipos que desarrollan la enfermedad de forma muy leve, es posible que sea una estimación baja con respecto a la real. Se trata de una enfermedad que afecta a diversos grupos étnicos y tanto a hombres como a mujeres, tras el nacimiento, las manifestaciones clínicas son muy diversas; es muy frecuente la hipoglicemia, que se relaciona con hiperplasia de los islotes de Langerhans del páncreas. ${ }^{4}$ También la macroglosia es un signo característico, pudiendo causar dificultad en la alimentación e incluso obstrucción de la vía aérea. .,11,2 Otras malformaciones asociadas pueden ser hipoplasia infraorbitaria, fisura palatina, criptorquidia y cardiopatías congénitas diversas.

Por esta variabilidad clínica, el diagnóstico puede plantear dificultades. En la literatura sólo Elliot y cols ${ }^{2}$ han establecido criterios diagnósticos: Criterios mayores: macroglosia, defectos de la pared abdominal (onfalocele, hernia umbilical, diástasis de los rectos abdominales) y peso prenatal y/o postnatal superior al P90. Criterios menores: signos auriculares característicos, nevus flammeus facial, hipoglicemia, nefromegalia y hemihipertrofia.

El diagnóstico se haría con la asociación de los tres criterios mayores; o de dos criterios mayores y tres menores. En este caso, no se pudo realizar estudio citogenético; sin embargo, el paciente reúne las características descritas en la literatura médica para ser diagnosticado como SBW; presenta tres criterios mayores: macroglosia, defectos de la pared abdominal tipo onfalocele y peso elevado al nacimiento (4000g. superior a P: 90) y un menor (hipoglucemia neonatal (glicemia50mg/dl).

La hipoglicemia se presenta hasta en un 50\% de los casos, se relaciona con hiperplasia de los islotes de Langerhans del páncreas. ${ }^{12}$ Hussain y cols. Reportaron un caso de SBW donde se evidenció la presencia de mosaico para disomía uniparental paterna del cromosoma 11p15, donde la persistencia del hiperinsulinismo estuvo asociada a pérdida de la función de los canales de K-ATP de las células $\beta$ pancreáticas. ${ }^{7}$

En la mayoría de los casos, la hipoglicemia puede ser transitoria e incluso asintomática y resolverse en los primeros días de vida; sin embargo, en el $5 \%$ de los casos puede persistir y extenderse hasta después del período neonatal, requiriendo alimentación continua y tratamiento médico como diazóxido, octreotide, glucagón, adrenalina e hidrocortisona; ${ }^{7,13}$ en raras ocasiones puede requerirse la pancreatectomía parcial, con resultados satisfactorios y disminución de la respuesta aguda de la insulina al estímulo con leucina y la prueba con glucosa, asociado con la reducción de la masa pancreática. ${ }^{5,13}$

En el caso clínico descrito, la hipoglicemia fue de inicio temprano (3 días de vida) requiriendo tratamiento con dextrosa endovenosa (infusión 
de glucosa $6 c c / \mathrm{kg} / \mathrm{min}$ ) y alimentación continua; con respuesta satisfactoria; en valoraciones sucesivas se evidencia euglicemia, con controles rigurosos de glicemias capilares, se concluye que se trató de una hipoglicemia transitoria.

TRATAMIENTO.

Los pacientes con hipoglicemia se pueden tratar con líquidos suministrados vía parenteral. Puede ser necesario reparar los defectos de la pared abdominal. Al niño se le debe realizar un cuidadoso seguimiento para vigilar la aparición de tumores. ${ }^{14}$

En el paciente se procedió a corregir la hipoglicemia transitoria con infusión de glucosa $6 \mathrm{cc} / \mathrm{kg} / \mathrm{min}$, intervención quirúrgica en dos tiempos. Reducción de onfalocele, malrotación completa de asas intestinales, laparoplastia, cierre de ileostomía, yeyunostomía.

\section{EXPECTATIVAS (PRONÓSTICO)}

Los niños con el síndrome de BeckwithWiedemann que sobreviven al período de la lactancia tienen buen pronóstico, aunque no se dispone de información sobre un seguimiento a largo plazo de la enfermedad. El desarrollo mental parece ir de normal a ligeramente disminuido. La hinchazón de la lengua puede causar problemas con la alimentación y el sueño. ${ }^{15}$ Las complicaciones neonatales son: apnea, cianosis, convulsiones, hipoglucemia, policitemia y trastornos respiratorios.

\section{CONCLUSIÓN}

El caso clínico descrito se trata de SBW, por presentar tres criterios mayores: onfalocele (defectos de la pared abdominal), macrosomía, macroglosia. y un criterio menor: hipoglicemia de inicio temprano y transitorio, con evolución clínica favorable; es de suma importancia la historia clínica y los criterios referidos en la literatura para no subestimar el diagnóstico al momento del nacimiento; actuar oportunamente, disminuir la mortalidad, secuelas y complicaciones, realizar un seguimiento riguroso por procesos neoplásicos a mediano - largo plazo, y mejorar de esta forma la calidad de vida.

\section{REFERENCIAS BIBLIOGRÁFICAS}

1. R. PARDO DE LA VEGA, et a 12001 . Caso Clínico Síndrome de Beckwith-Wiedemann: Factor de riesgo para el desarrollo de tumores. A propósito de un caso BOL PEDIATRIA: 41-44 Servicio de Pediatría. Hospital Central de Asturias.Oviedo

2. Elliot M, Bayly R, Cole T, Temple IK, Maher ER. Clinical features and natural history of BeckwithWiedemann syndrome. AM J Med Genet 1995; 56: 366-73

3. Fanaroff-Martin. "Enfermedades del feto y el recién nacido.” 5ta Edición. La Habana: Ed. CientíficoTécnica; 1985. Pp.1038-40.

4. Olivo Y, Flores N. Síndrome de Becwith-Wiedeman: Apropósito de un caso reportado en el servicio de neonatología del Hospital de Apoyo San José del Callao. Revista diagnóstico 2002; 41:76-79

5. Zaira Quijada et al 2006 Unidad de Endocrinología, Manifestaciones clínicas y paraclínicas en el Síndrome de Beckwith-Wiedemann caso clínico. Rev. Venez Endocrinol Metab 2006; 4 (2 Unidad de Endocrinología; 2 Unidad de Cirugía Pediátrica; IAHULA-ULA. Mérida, Venezuela

6. Sola A, Rogido M. "Cuidados Especiales del Feto y del Recién Nacido.” 2a ed, Vol. 1. Buenos Aires: Marcelo T. De Alvear Interamericana; 2001. p.192.

7. DeBaun MR, King AA, White N. Hypoglycemia in Beckwith-Wiedemann. Semin Perinatol 2000; 24:164-171.

8. Cuellar GO, Franco GM, González LD, Sandoval AM. Defectos de pared abdominal. Onfalocele y gastrosquisis. En: Jasso Gutiérrez Luis. Temas de Pediatría. Asociación Mexicana de Pediatría A.C. Neonatología. México: Interamericana McGrawHill, Inc; 1996: 179-189.

9. García H, Chávez A, Villegas S, Franco G, Alamilla $\mathrm{X}$. Morbilidad y mortalidad en recién nacidos con defectos de pared abdominal anterior (onfalocele y gastrosquisis). Gac Méd Méx 2002; 138: 519-526.

10. Malformaciones congénitas. Celostomía media u onfalocele. Gastrosquisis. En: Jasso Luis. Neonatología Práctica. 5a edición. México D.F.: Manual Moderno; 2002: 472-474.

11. Giancotti A, Romanini G, Docimo R, Arcuri C. (2003)," Clinical treatment of oral manifestations of Beckwith-Wiedeman syndrome in a child." J Clin Pediatr Dent, 27 pp. 377-80

12. Hussain K, Cosgrove KE, Shepherd RM, Luharia A, Smith VV, Kassem S, Gregory JW, Sivaprasadarao A, Chirstesen HT, Jacobsen BB, Brusgaard K, Glaser B, Maher EA, Lindley KJ, Hindmarsh P, Dattani M, Dunne MJ. Hyperinsulinemic hypoglycemia in BeckwithWiedemann syndrome due to defects in the function of pancreatic b-cell adenosine triphosphate-sensitive potassium channels. J Clin Endocrinol Metab 2005; 90:4376-4382. 
13. Munns CF, Batch JA. Hyperinsulinism and BeckwithWiedemann syndrome. Arch Dis Child Fetal Neonatal ED 2001; 84:F67-F69.

14. Cohen P, Hosono H. Hyperpituitarism, tall stature, and overgrowth syndromes. In: Kliegman, RM, Behrman RE, St. Geme III JW, Schor NF, Stanton BF, eds. Nelson Textbook of Pediatrics. 19th ed. Philadelphia, PA: Elsevier Saunders; 2011:chap 554.

15. Galerneau F. Beckwith-Wiedemann syndrome. In: Copel JA, D'Alton ME, Gratacós E, et al, eds.Obstetric Imaging. Philadelphia, PA: Elsevier Saunders; 2012:chap 111.

16. I. Arroyo Carrera1, Mํㅗㄹ L. Martínez-Frías2, J. Egüés Jimeno3, Mํㅗㅡ. García Martínez4, C. Eloína Cimadevilla Sánchez1, E. Bermejo Sánchez Síndrome de Wiedemann-Beckwith: Análisis clínico-epidemiológico de una serie consecutiva de casos en España - anales de pediatría española Vol. 50 № 2, 1999

17. Laura Pilar Martínez - 2006 (Enero-Marzo Macroglosia: Etiología multifactorial, manejo múltiple, O.D Vol. 37 № 1).
18. Orlando Jaramillo et al. Síndrome de BeckwithWiedemann: reporte del primer caso confirmado mediante bandeo cromosómico en Costa Rica cta Pediátrica Costarricense Copyright (C 1997, Asociación Costarricense de Pediatr

19. Niemitz EL, DeBaun MR, Fallon J, Murakami K, Kugo H, Oshimura M, Fenberg AP. Microdeletion of LIT1 in familial Beckwith-Wiedemann syndrome. Am J Hum Genet. 2004; 75:844-849

20. Dr. P. Lapunzina Badía.(2006) Guía clínica para el seguimiento de pacientes con síndrome de Beckwith-Wiedemann Servicio de Genética Médica. Hospital Universitario La Paz. P. ํ de la Castellana, 261. 28046. An Pediatr (Barc) 2006;64(3):252-9. 